\title{
Modelling magnetars' high energy emission through Resonant Cyclotron Scattering
}

\author{
Diego Götz* \\ CEA Saclay - DSM/Irfu/Service d'Astrophysique UMR AIM - Orme des Merisiers, Bat. 709, \\ F-91191 Gif-sur-Yvette, France \\ E-mail: diego.gotz@cea.fr
}

\section{Nanda Rea}

University of Amsterdam, Astronomical Institute Anton Pannekoek, Kruislaan, 403, 1098 SJ, Amsterdam, The Netherlands

\section{Silvia Zane}

${ }^{\dagger}$ Mullard Space Science Laboratory, University College London, Holmbury St. Mary, Dorking, Surrey, RH5 6NT, UK

\section{Roberto Turolla ${ }^{\dagger}$}

Department of Physics, University of Padova, Via Marzolo 8, I-35131 Padova, Italy

\section{Maxim Lyutikov}

Department of Physics, Purdue University, 525 Northwestern Avenue, West Lafayette, IN 47907, USA

\begin{abstract}
We present a systematic fit of a model of resonant cyclotron scattering (RCS) to the $\mathrm{X}$ and soft $\gamma$-ray data of four magnetars, including anomalous X-ray pulsars, and soft gamma repeaters. In this scenario, non-thermal magnetar spectra in the soft X-rays result from resonant cyclotron scattering of the thermal surface emission by hot magnetospheric plasma. We find that this model can successfully account for the soft X-ray emission of magnetars, while an additional component is still required to model the hard X-ray persistent magnetar emission recently discovered by INTEGRAL. The latter is an important component in terms of magnetars' luminosity, and cannot be neglected when modelling the soft X-ray part of the spectrum.
\end{abstract}

7th INTEGRAL Workshop

September 8-11 2008

Copenhagen, Denmark

\footnotetext{
${ }^{*}$ Speaker.
} 


\section{Introduction}

Soft Gamma Repeaters (SGRs) and Anomalous X-ray Pulsars (AXPs) (see [1] for a recent rewiev) are believed to be Magnetar candidates, namely isolated neutron stars powered by the decay of a huge $\left(B>B_{\text {crit }} \simeq 4.4 \times 10^{13} \mathrm{G}\right)$ magnetic fi eld [2]. They are slow rotators $(P \sim 2-12$ s), rapidly spinning down $\left(\dot{P} \sim 10^{-(11-12)} \mathrm{s} \mathrm{s}^{-1}\right)$, with rather soft (at least for AXPs) X-ray spectra that can be modelled by the sum of a black body $(k T \sim 0.5 \mathrm{keV})$ and a power law component $(\Gamma \sim$ $2-4)$. Some of them are transients, and they sporadically emit short ( $\sim 0.1 \mathrm{~s})$ bursts of high-energy radiation during unpredictable periods of activity. SGRs also emit giant flares: very powerful short spikes ( $E>10^{44} \mathrm{erg}$ ), followed by pulsating tails lasting a few hundreds of seconds. On the other hand the emission of short bursts by AXPs is sometimes associated with large flares in the persistent emission lasting hundreds of days, when the flux can vary over more than two orders of magnitudes.

As mentioned before, X-ray (0.1-10 keV) spectra of AXPs and SGRs are well described by an empirical model composed by the sum of a black body and a power law modifi ed by absorbtion. But as soon as the first persistent counterparts of magnetars have been reported at high energies ( $>20 \mathrm{keV}$ ) by the INTEGRAL observations (e.g. [3]), it was evident that this simple model was not adapted to describe the broad-band persistent spectra of Magnetars. Indeed, the INTEGRAL data showed the presence in fi ve sources of hard tails up to $\sim 200 \mathrm{keV}$ which lie well above the extrapolation of the X-ray data, especially for AXPs. This shows that at least some magnetars emit as much as, or even more, energy at hard X-rays than below $10 \mathrm{keV}$. Stimulated by this, and by the necessity of giving a physical interpretation of the two components at low energies, we implemented a simplifi ed Resonant Cyclotron Scattering model and we fi tted all magnetar spectra for which good quality XMM-Newton and INTEGRAL data are available. Our detailed results are reported in [4]. Here we focus only on the sources for which INTEGRAL data are available.

\section{Resonant Cyclotron Scattering}

Before discussing our XSPEC model and the implications of our results, we briefly touch on some properties of the RCS model which directly bear to the physical interpretation of the fitting parameters and their comparison with similar parameters introduced in other theoretical models. The basic idea follows the original suggestion by [5] (hereafter TLK), who pointed out that a scattering plasma may be supplied to the magnetosphere by plastic deformations of the crust, which twist the external magnetic field and push electric currents into the magnetosphere. The particle density of charge carries required to support these currents may largely exceed the Goldreich-Julian charge density [6]. Furthermore, it is expected that instabilities heat the plasma.

Following this idea, [7] studied how magnetospheric plasma might distort the thermal X-ray emission emerging from the star surface through effi cient resonant cyclotron scattering. If a large volume of the neutron star magnetosphere is filled by a hot plasma, the thermal (or quasi-thermal) cooling radiation emerging from the star surface will experience repeated scatterings at the cyclotron resonance. The effi ciency of the process is quantifi ed by the scattering optical depth, $\tau_{e s}$,

$$
\tau_{\text {res }}=\int \sigma_{\text {res }} n_{e} d l=\tau_{0}\left(1+\cos ^{2} \alpha\right)
$$


where

$$
\sigma_{\text {res }}=\frac{\sigma_{T}}{4} \frac{\left(1+\cos ^{2} \alpha\right) \omega^{2}}{\left(\omega-\omega_{B}\right)^{2}+\Gamma^{2} / 4}
$$

is the (non-relativistic) cross-section for electron scattering in the magnetized regime, $n_{e}$ is the electron number density, $\alpha$ is the angle between the photon propagation direction and the local magnetic field, $\Gamma=4 e^{2} \omega_{B}^{2} / 3 m_{e} c^{3}$ is the natural width of the fi rst cyclotron harmonic, $\sigma_{T}$ is the Thomson scattering cross-section, and

$$
\tau_{0}=\frac{\pi^{2} e^{2} n_{e} r}{3 m_{e} c \omega_{B}}
$$

Here $r$ is the radial distance from the center of the star, $\omega_{B}=e B / m_{e} c$ is the electron cyclotron frequency, and $B$ is the local value of the magnetic fi eld. At energies corresponding to soft X-ray photons, the resonant scattering optical depth greatly exceeds that for Thomson scattering, $\tau_{T} \sim$ $n_{e} \sigma_{T} r$,

$$
\frac{\tau_{r e s}}{\tau_{T}} \sim \frac{\pi}{8} \frac{m_{e} c^{3}}{e^{2} \omega_{B}} \sim 10^{5}\left(\frac{1 \mathrm{keV}}{\hbar \omega_{B}}\right) .
$$

This implies that even a relatively small amount of plasma present in the magnetosphere of the NS may considerably modify the emergent spectrum.

The RCS model developed by [7], and used in this investigation, is based on a simplifi ed, 1D semi-analytical treatment of resonant cyclotron up-scattering of soft thermal photons, under the assumption that scattering occurs in a static, non-relativistic, warm medium and neglecting electron recoil. The latter condition requires $\hbar \omega \ll m_{e} c^{2}$. Emission from the neutron star surface is treated assuming a blackbody spectrum, and that seed photons propagate in the radial direction. Magnetospheric charges are taken to have a top-hat velocity distribution centered at zero and extending up to $\pm \beta_{T}$. Such a velocity distribution mimics a scenario in which the electron motion is thermal (in $1 \mathrm{D}$ because charges stick to the fi eld lines). In this respect, $\beta_{T}$ is associated to the mean particle energy and hence to the temperature of the $1 \mathrm{D}$ electron plasma. Since scatterings with the magnetospheric electrons occur in a thin shell of width $H \sim \beta_{T} r / 3 \ll r$ around the "scattering sphere", one can treat the scattering region as a plane-parallel slab. Radiation transport is tackled by assuming that photons can only propagate along the slab normal, i.e. either towards or away from the star. Therefore, $\cos \alpha= \pm 1$ in eq. (2.1) and it is $\tau_{\text {res }}=2 \tau_{0}$; the electron density is assumed to be constant through the slab. We notice that the model does not account for the bulk motion of the charges. This is expected since the starting point is not a self-consistent calculation of the currents but a prescription for the charge density. As a consequence, the electron velocity and the optical depth are independent parameters, although in a more detailed treatment this might not be the case [8].

Although Thomson scattering conserves the photon energy in the electron rest frame, the (thermal) motion of the charges induces a frequency shift in the observer frame. However, since our electron velocity distribution averages to zero, a photon has the same probability to undergo up or down-scattering. Still, a net up-scattering (and in turn the formation of a hard tail in the spectrum) is expected if the magnetic fi eld is inhomogeneous. For a photon propagating from high to low magnetic fi elds, multiple resonant cyclotron scattering will, on average, up-scatter in energy 


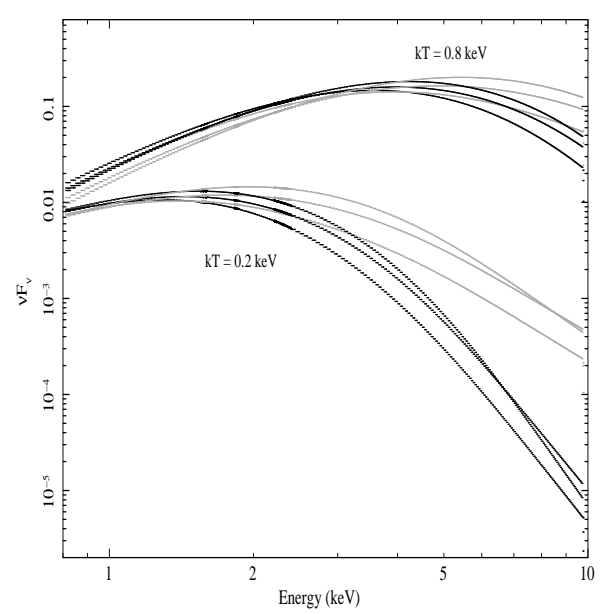

Figure 1: Distorsion of a seed blackbody spectrum through resonant cyclotron scattering onto magnetosferic electrons, for two values of the blackbody temperature, $0.2 \mathrm{keV}$ and $0.8 \mathrm{keV}$. Black lines show the RCS model for $\beta_{T}=0.2$ and $\tau_{\text {res }}=2,4,8$ (from bottom to top), while grey lines are relative to $\beta_{T}=0.4$ and $\tau_{\text {res }}=2,4,8$ (from bottom to top). The normalizations of the various curves are arbitrary.

the transmitted radiation, while the dispersion in energy decreases with optical depth [7]. Photon boosting by particle thermal motion in Thomson limit occurs due to the spatial variation of the magnetic fi eld and differs qualitatively from the (more familiar) non-resonant Comptonization [9]. As a result, the emerging spectrum is non-thermal and under certain circumstances can be modeled with two-component spectral models consisting of a blackbody plus a power-law [7].

In order to implement the RCS model in XSPEC, we created a grid of spectral models for a set of values of the three parameters $\beta_{T}, \tau_{\text {res }}$ and $T$. The parameter ranges are $0.1 \leq \beta_{T} \leq 0.5$ (step $0.1 ; \beta_{T}$ is the thermal velocity in units of $c$ ), $1 \leq \tau_{\text {res }} \leq 10$ (step 1 ; tau res is the optical depth) and $0.1 \mathrm{keV} \leq T \leq 1.3 \mathrm{keV}$ (step $0.2 \mathrm{keV} ; T$ is the temperature of the seed thermal surface radiation, assumed to be a blackbody). For each model, the spectrum was computed in the energy range $0.01-10 \mathrm{keV}$ (bin width $0.05 \mathrm{keV}$ ). The fi nal XSPEC at able spectral model has therefore three parameters, plus the normalization constant, which are simultaneously varied during the spectral fi tting following the standard $\chi^{2}$ minimization technique. In Fig. 1 we show the comparison between a blackbody model and our RCS model. We stress again that our model has the same number of free parameters (three plus the normalization) than the blackbody plus power-law or two blackbody models $\left(\beta_{T}, \tau_{\text {res }}, T\right.$, plus the normalization, compared to $k T, \Gamma$ (or $k T_{2}$ ), plus two normalizations); it has then the same statistical signifi cance. We perform in the following section a quantitative comparison between the RCS model and other models commonly used in the soft $\mathrm{X}$-ray range. However, note that here the RCS model is meant to model spectra in the $0.1-10 \mathrm{keV}$ energy range. For all sources with strong emission above $\sim 20 \mathrm{keV}$, the spectrum was modeled by adding to the RCS a power-law meant to reproduce the hard tail (see $\S 3$ for details). This powerlaw does not have (yet) a clear physical meaning in our treatment, but since it contributes also to the 0.1-10 keV band, our RCS parameters depend on the correct inclusion of this further component. 


\section{Results}

The complete dataset, used in [4], contains a set of AXPs which emit in the hard X-ray range, and also happen to be "steady" emitters or showing moderate flux and spectral variability (flux changes less than a factor of 5), and a group with larger variations: 4U 0142+614, 1RXS J17084009, 1E 1841-045, and 1E 2259+586. Then a set of "transient" AXPs (often labeled TAXPs), which includes XTEJ1810-197, 1E 1547.0-5408, and CXOU J1647-4552, was considered. To these we added 1E 1048-5937, in the light of the recent detection of large outbursts from this source (e.g. [10]), and of its spectral similarities with canonical TAXPs. Finally, a set of SGRs, which comprises SGR 1806-20, and SGR 1900+14 .

Here we report in detail only the results of the fi ts concerning the fi ve magnetars that have been fi rmly detected with INTEGRAL. For a complete description of the observations, as well as for data analysis techniques, see [4].

\subsection{AXPs: the hard X-ray emitters}

In this section we first consider the AXPs with INTEGRAL detected hard X-ray emission, which also coincides with the marginally variable AXPs. We recall that, strictly speaking, these hard X-ray emitting AXPs are not "steady" X-ray emitters. Subtle flux and spectral variability was discovered in 1RXS J1708-4009 and 4U 0142+614. In particular, 1RXS J1708-4009 showed a long term, correlated intensity-hardness variability (both in the soft and hard X-rays), most probably related to its glitching activity (e.g. [13]). 4U 0142+614 showed a flux increase of $\sim 10 \%$ (also correlated with a spectral hardening) following the discovery of its bursting activity [15]. Furthermore, thanks to a large RXTE monitoring campaign, long-term spin period variations and glitches were discovered in 4U 0142+614 1RXS J1708-4009, and 1E 1841-045, i.e. the three AXPs which are the brightest both in the soft and hard X-ray bands [16, 17, 15, 13].

Since these flux variations are rather small, we have chosen to model only the XMM-Newton observation closest to the INTEGRAL one (for 1RXS J1708-4009 only one XMM-Newton observation is available though). Our results from the spectral modeling of the $1-200 \mathrm{keV}$ spectrum of 4U 0142+614, 1RXS J1708-4009, and 1E 1841-045 are summarized in Table 1 and shown in Figs. 2 and 3. 
Table 1: Spectral Parameters: 4U 0142+614, 1RXS J1708-4009, 1E 1841-045, and SGR 1900+14

\begin{tabular}{|c|c|c|c|c|c|c|c|c|}
\hline \multirow{2}{*}{$\begin{array}{l}\text { Source Name } \\
\text { Parameters }\end{array}$} & \multicolumn{2}{|c|}{$4 \mathrm{U} 0142+614^{*}$} & \multicolumn{2}{|c|}{ 1RXS J1708-4009* } & \multicolumn{2}{|c|}{ 1E 1841-045 } & \multicolumn{2}{|c|}{ SGR 1900+14 } \\
\hline & $\mathrm{BB}+2 \mathrm{PL}$ & $\mathrm{RCS}+\mathrm{PL}$ & $\mathrm{BB}+2 \mathrm{PL}$ & $\mathrm{RCS}+\mathrm{PL}$ & $\mathrm{BB}+\mathrm{PL}$ & $\mathrm{RCS}+\mathrm{PL}$ & $\mathrm{BB}+\mathrm{PL}$ & $\mathrm{RCS}+\mathrm{PL}$ \\
\hline $\mathrm{N}_{H}$ & $1.67_{-0.02}^{+0.02}$ & $0.81_{-0.05}^{+0.05}$ & $1.91_{-0.06}^{+0.06}$ & $1.67_{-0.05}^{+0.05}$ & $2.38_{-0.1}^{+0.4}$ & $2.57_{-0.15}^{+0.13}$ & $3.5_{-0.1}^{+0.1}$ & $4.0_{-0.1}^{+0.1}$ \\
\hline constant & 1.01 & 1.10 & 1.05 & 0.80 & 1.02 & 1.09 & 1.20 & 1.10 \\
\hline $\mathrm{kT}(\mathrm{keV})$ & $0.43_{-0.03}^{+0.03}$ & $0.30_{-0.05}^{+0.05}$ & $0.47_{-0.01}^{+0.01}$ & $0.32_{-0.05}^{+0.05}$ & $0.51_{-0.02}^{+0.03}$ & $0.39_{-0.05}^{+0.05}$ & $\Omega .45_{-0.04}^{+0.04}$ & $0.30_{-0.1}^{+0.08}$ \\
\hline BB norm & $8.7_{-0.5}^{+0.4} \times 10^{-4}$ & & $2.4_{-0.2}^{+0.1} \times 10^{-4}$ & & $2.4_{-0.3}^{+0.6} \times 10^{-4}$ & & $6.7_{-0.1}^{+0.1} \times 10^{-5}$ & \\
\hline$\Gamma_{1}$ & $4.14_{-0.04}^{+0.04}$ & & $2.70_{-0.08}^{+0.08}$ & & & & $\mapsto$ & \\
\hline $\mathrm{PL}_{1}$ norm & $0.30_{-0.08}^{+0.08}$ & & $0.016_{-0.004}^{+0.00}$ & & & & (1) & \\
\hline$\beta_{T}$ & & $0.33_{-0.05}^{+0.05}$ & & $0.38_{-0.03}^{+0.03}$ & & $0.23_{-0.05}^{+0.05}$ & & $0.26_{-0.03}^{+0.03}$ \\
\hline$\tau_{\text {res }}$ & & $1.9_{-0.2}^{+0.2}$ & & $2.1_{-0.2}^{+0.2}$ & & $1.13_{-0.2}^{+0.3}$ & & $2.5_{-0.2}^{+0.5}$ \\
\hline RCS norm & & $4.5_{-0.8}^{+0.6} \times 10^{-3}$ & & $8.1_{-1.3}^{+1.1} \times 10^{-4}$ & & $3.1_{-1.1}^{+2.3} \times 10^{-}$ & & $1.8_{-0.05}^{+0.04} \times 10^{-4}$ \\
\hline$\Gamma_{2}$ & $0.78_{-0.07}^{+0.1}$ & $1.1_{-0.1}^{+0.1}$ & $0.76_{-0.1}^{+0.1}$ & $1.0_{-0.1}^{+0.1}$ & $1.47_{-0.05}^{+0.04}$ & $1.47_{-0.05}^{+0.05}$ & $\infty \quad 1.4_{-0.1}^{+0.1}$ & $1.24_{-0.07}^{+0.07}$ \\
\hline $\mathrm{PL}_{2}$ norm & $1.4_{-0.1}^{+0.1} \times 10^{-4}$ & $5.0_{-0.1}^{+0.1} \times 10^{-4}$ & $8.6_{-0.1}^{+0.1} \times 10^{-5}$ & $4.2_{-0.1}^{+0.1} \times 10^{-4}$ & $2.4_{-0.6}^{+0.6} \times 10^{-3}$ & $2.2_{-0.1}^{+0.1} \times 10^{-}$ & $-34.4_{-0.1}^{+0.1} \times 10^{-4}$ & $3.0_{-0.1}^{+0.1} \times 10^{-4}$ \\
\hline $\begin{array}{l}\text { Flux } 1-10 \mathrm{keV} \\
\text { Flux } 1-200 \mathrm{keV}\end{array}$ & $\begin{array}{l}1.1_{-0.8}^{+0.8} \times 10^{-10} \\
2.3_{-1.1}^{+1.7} \times 10^{-10}\end{array}$ & $\begin{array}{l}1.1_{-0.8}^{+0.8} \times 10^{-10} \\
2.3_{-1.3}^{+1.0} \times 10^{-10}\end{array}$ & $\begin{array}{l}2.6_{-0.3}^{+0.3} \times 10^{-11} \\
1.1_{-0.5}^{+0.5} \times 10^{-10}\end{array}$ & $\begin{array}{l}2.6_{-0.8}^{+1.1} \times 10^{-11} \\
1.4_{-0.8}^{+0.8} \times 10^{-10}\end{array}$ & $\begin{array}{l}2.2_{-0.3}^{+0.2} \times 10^{-11} \\
1.1_{-0.8}^{+0.8} \times 10^{-10}\end{array}$ & $\begin{array}{l}2.1_{-0.3}^{+0.2} \times 10^{-} \\
1.1_{-0.6}^{+0.8} \times 10^{-}\end{array}$ & $\begin{array}{l}-113.9_{-0.1}^{+0.1} \times 10^{-12} \\
-101.7_{-0.1}^{+0.1} \times 10^{-11}\end{array}$ & $\begin{array}{l}3.8_{-0.1}^{+0.1} \times 10^{-12} \\
1.7_{-0.1}^{+0.1} \times 10^{-11}\end{array}$ \\
\hline$\chi_{\nu}^{2}(\mathrm{dof})$ & $0.99(216)$ & $0.80(216)$ & $1.11(202)$ & $1.01(202)$ & $1.14(158)$ & $1.08(156)$ & $1.18(141)$ & $1.15(139)$ \\
\hline
\end{tabular}

Best fit values of the spectral parameters obtained by fitting the $\sim 1-200 \mathrm{keV}$ XMM-Newton and INTEGRAL AXPs' spectra with a blackbody plus two power-laws model (BB+2PL) for 4U 0142+614 and 1RXS J1708-4009, while a single power-law was used for 1E 1841-045 and SGR 1900+14. Furthermore, all the sources were modeled with a resonant cyclotron scattering model plus a power-law (RCS+PL). Errors are at $1 \sigma$ confidence level, reported fluxes are absorbed and in units of erg s${ }^{-1} \mathrm{~cm}^{-2}$, and $\mathrm{N}_{H}$ in units of $10^{22} \mathrm{~cm}^{-2}$ and assuming solar abundances from[18]; 2\% systematic error has been included. See also Figs. 2, 3, 4 and $\S 3.1$ for details. ${ }^{*}$ : source slightly variable in flux and spectrum, see text for details. 
In all cases we found that $N_{H}$, as derived from the RCS model, is lower than (or consistent with) that inferred from the BB+2PL fit (or BB+PL in the case of 1E 1841-045), and consistent with what derived from fi tting the single X-ray edges of 4U 0142+614, and 1RXS J1708-4009 [14]. This is not surprising, since the power-law usually fi tted to magnetar spectra in the soft X-ray range is well known to cause an overestimate in the column density ${ }^{1}$ The surface temperature we derived fitting the RCS model is systematically lower than the corresponding BB temperature in the $\mathrm{BB}+2 \mathrm{PL}$ or $\mathrm{BB}+\mathrm{PL}$ models, and is consistent with being the same $(\sim 0.33 \mathrm{keV})$ in the four sources. On the other hand the thermal electron velocity and the optical depth are in the ranges $0.2-0.4$ and 1.0-2.1, respectively. Concerning the hard X-ray power-law, we find that the photon index is, within the errors, the same when fi tting the RCS or the BB+2PL or BB+PL models, while the hard PL normalization is larger in the RCS case with respect to the BB+2PL model. Both the soft and the hard X-ray fluxes of all these AXPs derived from the RCS fi tting are consistent with those implied by the usual BB+2PL fi tting.

\subsection{SGRs}

Finally, we consider the $1-200 \mathrm{keV}$ emission of SGRs (see Table 1 and Fig. 4). It has been already noticed that the hard X-ray emission of SGRs is quite different from that of AXPs [3]. In fact, the spectra of AXPs show a clear turnover between 10 and $20 \mathrm{keV}$ (see Fig. 2 and 3) and the fi $t$ requires an additional spectral component. Instead, the hard X-ray emission of SGRs seems the natural continuation of the non-thermal component which is dominant in the $1-10 \mathrm{keV}$ energy range. This is why we can use a BB (or RCS) plus a single power-law in the entire $1-200 \mathrm{keV}$ range, while for the hard X-ray emitting AXPs we were forced to add a second power-law to the BB+PL model.

Despite the fact that SGR 1806-20 and SGR 1900+14 are both detected by INTEGRAL, here we report the results on SGR 1900+14 only, since SGR 1806-20 has been proven to be variable an time scales which are shorter than the ones required by INTEGRAL/IBIS in order to obtain a good quality spectrum, and hence we are not able to deal with a truly simultaneous broad band spectrum.

In the SGR 1900+14 1-200 keV spectrum, we found consistent $N_{H}$ and spectral index values between the $\mathrm{BB}+\mathrm{PL}$ and RCS+PL models, and a RCS surface temperature signifi cantly lower than the corresponding BB temperature. In all the SGR observations, the derived fluxes are consistent among the two models.

\section{Discussion}

Before discussing our results and the physics we can derive from our model, we would like to stress once again that the RCS model involves a number of simplifi cations (see $\$ 2$ ). One is the assumption of a single temperature surface emission. Current-carrying charges will hit and heat the star surface, generally inhomogeneously (TLK). In addition, the emission emerging from the surface is likely to be non-Planckian. While the presence of an atmosphere on top the crust of a magnetar remains a possibility $[19,20]$, its properties, are then likely different from those of a

\footnotetext{
${ }^{1}$ This is because the absorption model tends to increase the $N_{H}$ value in response of the steep rise of the power-law at low energies, which eventually diverges approaching $\mathrm{E}=0$.
} 

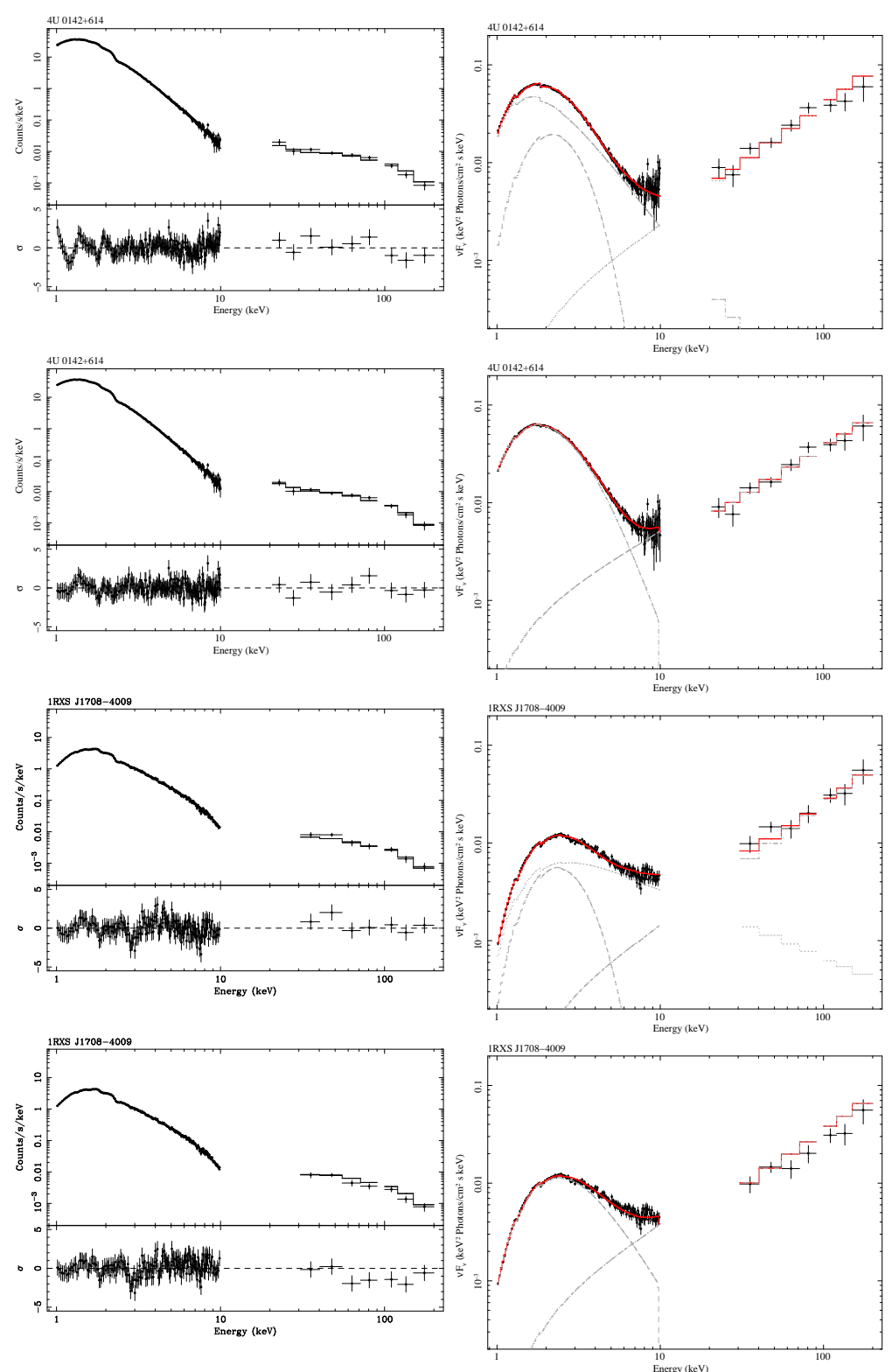

Figure 2: 4U 0142+614, and 1RXS J1708-4009: left column shows the spectra in Counts/s/keV while in the right column we report the $\nu \mathrm{F}_{\nu}$ plots. The upper panels are relative to the modeling with a blackbody plus two power-laws $(\mathrm{BB}+2 \mathrm{PL})$. Bottom panels report for both sources the resonant cyclotron scattering plus a power-law model (RCS+PL).

standard (in radiative and hydrostatic equilibrium) atmosphere on, e.g., a canonical isolated cooling neutron star (see e.g. [21]). The extreme fi eld and (relatively) low surface temperature $(\lesssim 0.5 \mathrm{keV})$ of magnetar candidates may also be suggestive of a condensed surface, at least if the chemical composition is mainly Fe [22]. In the light of these considerations, and in the absence of a detailed model for the surface emission, and for the atmosphere of strongly magnetized NSs constantly hit by returning currents, we restricted ourself to a blackbody approximation for the seed thermal photons. 
In spite of these simplifications, [4] found that the RCS model can describe the soft X-ray portion of the whole set of magnetar spectra we have considered there, including the TAXPs variability, by using only three free parameters (plus a normalization factor). This is the same number of degrees of freedom required by the blackbody plus power law model, commonly used to fi $t$ this energy band.

\subsection{Magnetar magnetospheric properties}

One of the most interesting outcomes of our analysis is the measure of the magnetospheric properties of magnetars. In all sources, steady and variable ones (see [4]), the value of $\tau_{\text {res }}$ is in the range of $\sim 1-6$. This suggests that the entire class of sources are characterized by similar properties of scattering electrons, their density and their (thermal) velocity spread. An optical depth $\tau_{0}=\tau_{\text {res }} / 2$ requires a particle density $n_{e}$ (see eq. [2.3]) which can be easily inferred considering:

$$
\tau_{0} \approx 1.8 \times 10^{-20} n_{e} r_{s c}\left(\frac{1 \mathrm{keV}}{\hbar \omega_{B}}\right)
$$

where $r_{s c}$ is the radius of the scattering sphere

$$
r_{s c} \approx 8 R_{N S}\left(\frac{B}{B_{\text {crit }}}\right)^{1 / 3}\left(\frac{1 \mathrm{keV}}{\hbar \omega_{B}}\right)^{1 / 3},
$$

$R_{N S}$ is the neutron star radius and $B_{\text {crit }} \approx 4.4 \times 10^{13} \mathrm{G}$ is the quantum critical fi eld. By taking a typical photon energy of $\sim 1 \mathrm{keV}, R_{N S} \sim 10^{6} \mathrm{~cm}$ and $B \sim 10 B_{\text {crit }}$, we get $n_{e} \approx 1.5 \times$ $10^{13} \tau_{\text {res }} \mathrm{cm}^{-3}$. This is several orders of magnitude larger than the Goldreich-Julian density [6] at the same distance, $n_{G J} \approx n_{e} \pi r_{s c} /\left(3 \tau_{\text {res }} R_{l c}\right) \sim 2 \times 10^{10} \mathrm{~cm}^{-3}$ (where $R_{l c}$ is the light cylinder
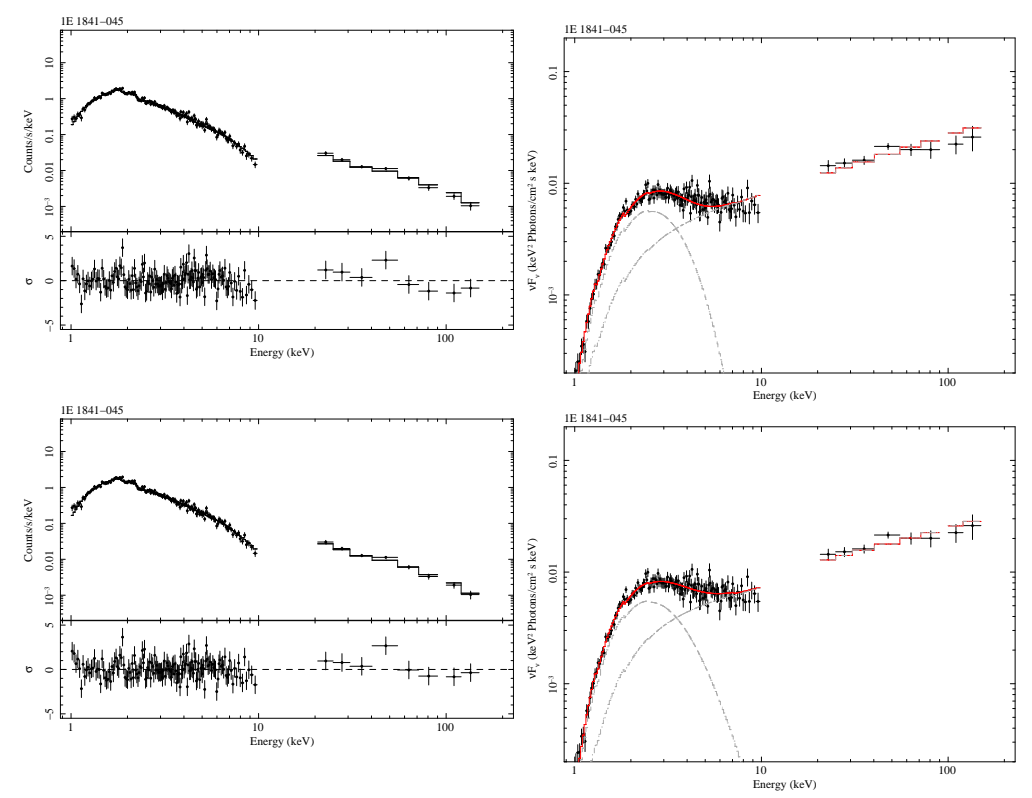

Figure 3: 1E 1841-045: left column shows the spectra in Counts $/ \mathrm{s} / \mathrm{keV}$ while in the right column we report the $\nu \mathrm{F}_{\nu}$ plots. The upper panels are relative to the modeling with a a blackbody plus power-law, while bottom panels report the resonant cyclotron scattering plus a power-law model (RCS+PL). 

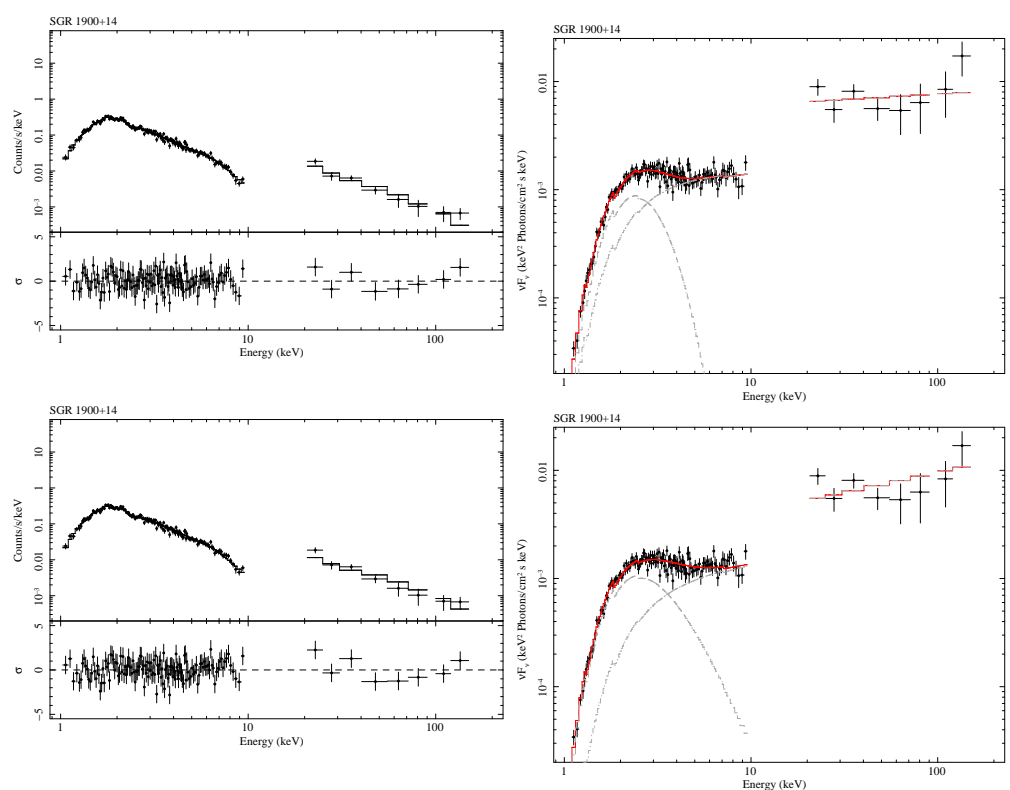

Figure 4: SGR 1900+14: left column shows the spectra in Counts/s/keV while in the right column we report the $\nu \mathrm{F}_{\nu}$ plots. The upper panels are relative to the modeling with a blackbody plus power-law (BB+PL), while bottom panels report the resonant cyclotron scattering model plus power-law (RCS+PL). The red lines represent the total model, while the dashed lines are the single components.

radius and we took $P \sim 10 \mathrm{~s}$ ). While the charge density is large when compared with the minimal Goldreich-Julian density, it provides a negligible optical depth to non-resonant Thomson scattering. Only the resonant cyclotron scattering makes an effi cient photon boosting possible.

Our present model does not include a proper treatment of magnetospheric currents, so that $\tau_{\text {res }}$ is a free parameter related to the electron density. Nevertheless, it is useful to compare the values of the optical depth inferred here to those expected when a current flow arises because a steady twist is implanted in the star magnetosphere, as in the case investigated by TLK under the assumption of axisymmetry and self-similarity. If the scattering particles have a collective motion (bulk velocity $\beta_{\text {bulk }}$ ), the effi ciency of the scattering process is related to $\tau_{\text {res }} \beta_{\text {bulk }}$ (e.g. [23]). This quantity is shown as a function of the magnetic colatitude in Fig. 5 of TLK for different values of the twist angle, $\Delta \phi_{N-S}$. By assuming $\beta_{b u l k}=1$ and integrating over the angle, we get the average value of the scattering depth as a function of $\Delta \phi_{N-S}$. A value of $\tau_{r e s} \sim 1$ is only compatible with very large values of the twist angle (i.e $\Delta \phi_{N-S}>3$ ), while typical values of $\tau_{\text {res }} \sim 2$, as those obtained from some of our fi ts, require $\beta_{b u l k} \lesssim 0.5$ to be compatible with $\Delta \phi_{N-S} \sim 3$ (the smaller is $\beta_{b u l k}$, the smaller is the value of the twist angle). This is consistent with the fact that the RCS model has been computed under the assumption of vanishing bulk velocity for the magnetospheric currents, and it is compatible with TLK model only when in the latter it is $\beta_{b u l k} \ll 1$.

\section{Conclusions}

In the last few years the detection of bursts from AXPs strengthened their connection with SGRs. However, the latter behave differently in many respects. Below $\sim 4 \mathrm{keV}$, the SGRs emission can be described either by a blackbody or an RCS component. At higher energies though $(>4 \mathrm{keV})$, 
their spectra require the addition of a power-law component, which well describes the spectrum until $\sim 200 \mathrm{keV}$. The non-thermal component dominates their spectra to the point that the choice of a blackbody or the RCS model at lower energies does not affect signifi cantly the value of the hard $\mathrm{X}$-ray power-law index, nor the energy at which this component starts to dominate the spectrum. The spectra of SGRs are then strongly non-thermally dominated in the 4-200 keV range.

The case of the AXPs is different (with the exception of 1E 1841-045). These sources show a more complex spectrum, with an evident non-thermal component below $\sim 10 \mathrm{keV}$, apparently different from that observed at higher energies. For the AXPs detected at energies $>20 \mathrm{keV}$, the spectrum can be described by a RCS component until 5-8 keV, above which the non-thermal hard X-ray component becomes important, and (e.g. for 1RXS J1708-4009 and 4U 0142+614) dominates until $\sim 200 \mathrm{keV}$. In the case of the $\mathrm{BB}+2 \mathrm{PL}$ model instead, the non-thermal component responsible for the hard X-ray part of the spectrum starts to dominate only above $\sim 10 \mathrm{keV}$ (see e.g. Figs. 2 and 3). It is worth noting that the photon index of the hard X-ray component in AXPs does not strongly depend on the modeling of the spectrum below $10 \mathrm{keV}$, while, its normalization and, as a consequence, the value at which the hard tail starts to dominate the spectrum, do.

The fact that hard X-ray spectra detected from AXPs are much flatter than those of SGRs may also suggest a possible difference in the physical mechanism that powers the hard tail in the two classes of sources. Within the magnetar scenario, [24] discussed how soft $\gamma$-rays may be produced in a twisted magnetosphere, proposing two different pictures: either thermal bremsstrahlung emission from the surface region heated by returning currents, or synchrotron emission from pairs created higher up $(\sim 100 \mathrm{~km})$ in the magnetosphere. Moreover, a third scenario involving resonant magnetic Compton up-scattering of soft X-ray photons by a non-thermal population of highly relativistic electrons has been proposed by [25]. It is interesting to note that 3D Monte Carlo simulations [26, 27] show that multiple peaks may appear in the spectrum. In particular, in the model by [27], a second "hump" may be present when up-scattering is so effi cient that photons start to fill the Wien peak at the typical energy of the scattering electrons. The change in the spectral slope may be due, in this scenario, to the peculiar, "double-humped" shape of the continuum. The precise localization of a possible down-break at higher energies is therefore of great potential importance and might provide useful information on the underlying physical mechanism responsible for the hard emission.

\section{Acknowledgments}

D. G. aknowledges the French Space Agency (CNES) for fi nancial support. N.R. is supported by an NWO Veni Fellowship, and acknowledges the warm hospitality of the Mullard Space Science Laboratory, where this work was started, and of the Purdue University where it has been completed. S.Z. acknowledges STFC for support through an Advanced Fellowship. Based on observations with INTEGRAL and XMM-Newton, two ESA missions with instruments and science data centres funded by ESA member states with the participation of Russia and the USA. The RCS model is available to the community on the XSPEC website $^{2}$.

\footnotetext{
${ }^{2}$ http://heasarc.gsfc.nasa.gov/docs/xanadu/xspec/models/rcs.html
} 


\section{References}

[1] Mereghetti, S. 2008, A\&ARv, 225, 15

[2] Duncan, R., \& Thompson, C. 1992, ApJ, 392, L9

[3] Götz, D., Mereghetti, S., Tiengo, A., et al. 2006, A\&A, 449, L31

[4] Rea, N., Zane, S., Turolla, R., et al. 2008, ApJ, 686, 1245

[5] Thompson, C., Lyutikov, M. \& Kulkarni, S.R., 2002, ApJ, 574, 332

[6] Goldreich, P. \& Julian W.H. 1969, ApJ, 157, 869

[7] Lyutikov, M., \& Gavriil F.P., 2006, MNRAS, 368, 690

[8] Beloborodov, A. M. \& Thompson, C. 2007, ApJ, 657, 967

[9] Kompaneets, A.S., 1956, Sov. Phys. JETP, 31, 876

[10] Mereghetti, S., Tiengo, A., Stella, L., et al., 2004, ApJ, 608, 427

[11] Kaspi, V.M., Gavriil, F.P., Woods, et al., \& Chakrabarty, D., 2003, ApJ, 588, L93

[12] Woods, P.M., et al. 2004, ApJ, 605, 378

[13] Israel, G. L., Götz, D., Zane, S., Dall'Osso, S., Rea, N., Stella, L. 2007, A\&A, 476, L91

[14] Durant, M., \& van Kerkwijk, M. H., 2006, ApJ, 650, 1082

[15] Dib, R., Kaspi, V. M., Gavriil, F. 2008, ApJ, 673, 1044

[16] Gavriil, F.P. \& Kaspi, V.M. 2002, ApJ, 567, 1067

[17] Dall'Osso, S., Israel, G.L., Stella, L., Possenti, A., \& Perozzi, E. 2003, ApJ, 499, 485

[18] Lodders, K. 2003, ApJ, 591, 1220

[19] Güver, T., Özel, F., Gög̈üs, E., Kouveliotou, C., 2007, ApJ, 667, L73

[20] Güver, T., Özel, F., \& Gögüs, 2008, ApJ, 675, 1499

[21] Ho, W.C.G. \& Lai, D. 2003, MNRAS, 338, 233

[22] Turolla, R., Zane, S. \& Drake, J.J. 2004, ApJ, 603, 265

[23] Nobili, L., Turolla, R. \& Zampieri, L. 1993, ApJ, 404, 686

[24] Thompson, C., \& Beloborodov, A. M., 2005, ApJ, 634, 565

[25] Baring M.,G., \& Harding A.K. 2007, Ap\&SS, 308, 109

[26] Fernandez, R., \& Thompson, C., 2007, ApJ, 660, 615

[27] Nobili, L., Turolla, R. \& Zane, S., 2008, MNRAS, 386, 1527 